\title{
Incidentally Discovered Papillary Thyroid \\ Microcarcinoma in Patients Undergoing Thyroid Surgery for Benign Disease
}

Joaquin de Carlos ( $\sim$ decarlosjoaquin@gmail.com )

Hospital de Navarra: Complejo Hospitalario de Navarra https://orcid.org/0000-0001-5875-4524

Ander Ernaga

Hospital de Navarra: Complejo Hospitalario de Navarra

Ana Irigaray

Hospital de Navarra: Complejo Hospitalario de Navarra

Jose Javier Pineda

Hospital de Navarra: Complejo Hospitalario de Navarra

Ana Echegoyen

Hospital de Navarra: Complejo Hospitalario de Navarra

Pilar Salvador

Hospital de Navarra: Complejo Hospitalario de Navarra

Emma Anda

Hospital de Navarra: Complejo Hospitalario de Navarra

\section{Research Article}

Keywords: Benign thyroid disease, thyroid surgery, papillary thyroid microcarcinoma, incidental

Posted Date: March 21st, 2022

DOI: https://doi.org/10.21203/rs.3.rs-1229136/v2

License: (c) (i) This work is licensed under a Creative Commons Attribution 4.0 International License.

Read Full License 


\section{Abstract}

\section{Introduction}

Incidence of thyroid carcinoma (TC) has grown significantly over the last few decades worldwide, partly due to the increase detection of small thyroid microcarcinoma (TMc). TMc are tumors with a maximal diameter $\leq 1 \mathrm{~cm}$, identified during histopathology examination following a thyroidectomy performed for reasons not pertaining to malignancy. The aim of this study is to investigate the prevalence of papillary thyroid microcarcinoma (PTMc) according to the nature of benign pathology that submit patients to thyroid surgery and its trend evolution.

\section{Methods}

Retrospective cohort analysis of 1815 patients who underwent total thyroidectomy for non-malignant disease from 2005 to 2020.

\section{Results}

The mean age of subjects was 53.5 years, with a higher proportion of women (1481, 82.1\%). A total of 167 PTMc (9.3\%) were incidentally discovered. Multivariate logistic regression analysis shows no differences in prevalence according to sex or age in patients with PTMc compared to those with final benign histology. Multinodular goiter increases the risk of PTMc with an odds ratio of $2.2(p=0.001)$ compared to Hashimoto's thyroiditis and Graves' disease (GD). There is a statistically significant increase in the incidence of PTMc in the group operated between $2017-2020$ vs. 2005-2008 $(p=0.005)$

\section{Conclusion}

Overall prevalence of PTMc in patients who underwent thyroid surgery for benign disease was $9.3 \%$. Thyroid nodular hyperplasia was the most frequent benign pathology associated to this occult cancer as compared to Hashimoto or GD. Gender and age were not correlated with prevalence of TMc. Over the years, surgical findings of PTMc have grown, particularly in the 2017-2020 period.

\section{Introduction}

Thyroid carcinoma (TC) is responsible over $2 \%$ of all tumors, being considered the most frequent neoplasm of the endocrine system. Incidence has been rapidly increased in recent decades around many countries worldwide, with a female-to-male ratio of 3:1 (1). However, mortality has remained stable or even declining in most areas, at $0.6 / 100.000$ women and $0.3 / 100.000$ men per year (2). This phenomenon is partly due to overdiagnosis of small and subclinical tumors (3)(4). Approximately $90 \%$ are differentiated cancers originating from thyroid follicular cells, being papillary thyroid carcinoma (PTC) the most common histological subtype, followed by follicular thyroid carcinoma (5). 
Papillary thyroid microcarcinoma (PTMc) also called occult sclerosing carcinoma, occult papillary carcinoma and non-encapsulated sclerosing tumor, is a PTC defined as measuring 1 centimeter $(\mathrm{cm})$ or less (6). This carcinoma could be solitary or detected in multiple foci (7). The term incidental is used for tumors identified unexpectedly at the anatomopathological study after thyroidectomy carried out for benign reason or identified randomly while undergoing cervical examinations performed for another purpose (8). The majority of them are PTMc (9). Occult medullary thyroid cancer (MTC) is an uncommon entity with a prevalence of approximately $0.3 \%$, most of them are small and confined to the gland (10) (11). Occult carcinoma is significantly prevalent in population with a reported autopsy incidence of 0.5$36 \%$ (6). Due to his indolent evolution, it has been proposed to be renamed as "papillary microtumor" (6). Natural history and growth kinetics of the majority of clinical low risk intrathyroidal small PTC is excellent (12). As a result of their indolent evolution, they rarely or never progress (13).

We performed a study to define the prevalence of PTMc in patients undergoing total thyroidectomy for benign disease. Secondly, we aimed to detect whether there are different rates according to the type of underlying pathology: Graves' disease (GD), multinodular goiter (MNG), chronic lymphocytic thyroiditis (CLT). Finally, it is intended to detail the evolution of the number of surgeries and PTMc findings from 2005 to 2020.

\section{Materials And Methods}

All patients referred to thyroid surgery in a high-volume tertiary referral hospital, Hospital Universitario de Navarra (Pamplona, Spain) were enrolled. Inclusion criteria were patients who underwent total thyroidectomy between 2005 to 2020 with histologically confirmed papillary thyroid microcarcinoma (largest diameter $\leq 10 \mathrm{~mm}$ ) diagnosed incidentally in subjects undergoing thyroid surgery for benign disease. We exclude all patients with reported irradiation of head or neck, subjects with genetic syndromes predisposing to the development of PTC, operated for suspicion of nodular malignancy and histologic confirmation of TC with a diameter exceeding $1 \mathrm{~cm}$. Patients who underwent hemithyroidectomy and subjects with final histology that differs from PTMc were also excluded. Distant or nodal metastasis from thyroid cancer could not have been the reason for the surgical procedure.

Database recorded clinical, laboratory, imaging, indication for surgery, surgery performed, cytological and histopathological information. Patients were classified according to pathology for which the indication of surgery was performed: CLT, GD and MNG.

Presence of compressive symptoms was the indication of surgery in CLT and MNG. Operation was performed on GD in patients that require definitive treatment due to relapse or lack of response to antithyroid drugs. All patients had pre-surgical thyroid ultrasound, having performed cytological study of the dominant nodule if indicated. All those with results suspicious (Bethesda III, IV) or compatible with malignancy were excluded (Bethesda V, VI). Histological study was carried out by expert thyroid pathologists, carrying out a routine study of the thyroid nodules, capsular parenchyma and defining 
whether vascular invasion was present. The same standard protocol was used to examine the surgical specimen in all patients. Surgery was conducted by general surgeons with expertise in thyroid pathology.

SPSS software (version 16.0.2: SPSS, Inc., Chicago, Illinois, USA) was used to perform the statistical analysis of the registry. Kolmogorov-Smirnov test was carried out to ensure the normality of the data distribution. Categorical variables were expressed as number and frequencies and compared using chisquared test. Data is presented as mean - standard deviation. Proportions were analyzed with chisquared test, logistic regression with $95 \%$ confidence intervals $(\mathrm{Cl})$ were calculated. Continuous variables were compared using parametric or non-parametric test, as required. Additionally, we performed a multivariate logistic regression model to test the association of age, sex, CLT, MNG, GD and year of surgery with the development of PTMc. P-values of $<0.05$ were statistically significant. All tests were twosided.

\section{Results}

A total of 2920 consecutive patients who have undergone thyroid surgery for benign disease were enrolled. However, 1105 subjects were excluded: 618 with TC larger than $1 \mathrm{~cm}, 487$ who underwent hemithyroidectomy and 13 with histology different to PTMc. Finally, 1802 satisfied the inclusion criteria, flow chart of study is illustrated in Fig. 1. Overall papillary microcarcinoma prevalence in patients treated by total thyroidectomy for benign indication were 167 cases (9.3\%), classified as occult PTMc. We also detect microcarcinomas of other histological variants, such as 6 follicular carcinoma (3.3\%), 5 medullary $(2.8 \%), 1$ Hürthle $(0.6 \%)$ and 1 non-invasive follicular thyroid neoplasm with papillary like nuclear features (0.6\%) (NIFTP).

Baseline characteristics of the sample are presented in Table 1. Demographic and clinical features were analyzed to evaluate any significant associations comparing PTMc versus patients with benign disease (Table 2). In the overall univariate analysis, age and gender were not statistically associated with cancer. Median age of presentation of cases without PTMc was $53.5 \pm 12.9$ vs $55.5 \pm 13.9$ in non PTMc $(p=$ 0.970). The percentage of men that presented with PTMc was $21.5 \%$ (36), compared to $17.6 \%(287)$ without PTMc $(p=0.199)$. Nevertheless, cancer rates according to benign thyroid pathology for surgery were as follows: CLT 6.5\% (3/46), GD 4.9\% (17/349), MNG 10.5\% (147/1406). We noted a higher proportion of PTMc in MNG, resulting statistically significant $(p=0.001)$ vs CLT/GD. The four-year period prevalence of incidental PTMc and benign histology is shown in Fig. 2. It has been noted a progressively and significant increasing trend of diagnosed PTMc over the years $(p=0.010)$. 
Table 1

Demographic and clinical characteristics of the cohort.

\begin{tabular}{|ll|}
\hline Total population & 1802 \\
\hline Age at diagnosis, years & $53.5 \pm 13.8$ \\
(mean \pm standard deviation) & \\
\hline Sex, $\mathrm{n}(\%)$ : & \\
- Female (\%) & $1481(82.1 \%)$ \\
- Male (\%) & $321(17.9 \%)$ \\
\hline Benign indication for surgery: & \\
- Chronic lymphocytic thyroiditis & $46(2.5 \%)$ \\
- Graves' disease & $350(19.4 \%)$ \\
- Nodular hyperplasia & $1406(78.0 \%)$ \\
\hline Presence of incidental papilar microcarcinoma & $167(9,3 \%)$ \\
\hline
\end{tabular}


Table 2

Incidence of PTMc in relation to sex, age, benign thyroid disease, and time of diagnosis.

\begin{tabular}{|llll|}
\hline & Cases without PTMc & Cases with PTMc & p \\
\hline Age at diagnosis, years & $55.5 \pm 13.9$ & $53.5 \pm 12.9$ & 0.970 \\
(mean \pm standard deviation) & & & \\
\hline Age $\geq 55$ & $784(48.0)$ & $79(47.3)$ & 0.874 \\
\hline Sex: & & & 0.199 \\
- Male (\%) & $287(31.4 \%)$ & $36(21.6)$ & \\
- Female (\%) & $628(68.6 \%)$ & $131(78.4)$ & \\
\hline Benign indication for surgery: & & & 0.001 \\
- Chronic lymphocytic thyroiditis (\%) & $43(2.6)$ & $3(1.8)$ & \\
- Graves' disease (\%) & $332(20.3)$ & $17(10.2)$ & \\
- Multinodular goiter (\%) & $1259(77.1)$ & $147(88.0)$ & \\
\hline Surgery date: & & & \\
- 2005-2008 & $340(93.1)$ & $25(6.9)$ & \\
- 2009-2012 & $465(91.5)$ & $43(8.5)$ & \\
- 2013-2016 & $\mathbf{4 4 8 ( 9 1 . 6 )}$ & $\mathbf{4 1}(8.4)$ & \\
- 2017-2020 & $382(86.8)$ & $58(13.2)$ & \\
\hline
\end{tabular}

On multivariate analysis, we found no association with gender and age. By contrast, MNG disease was significantly associated with incidental thyroid cancer with an odds ratio of 2.2 (Cl 95\%, 1.4-3.5; $p=$ 0.001). This reflects a strength of the association between the development of PTMc in MNG. The risk of PTMc is $120 \%$ higher in MNG compared with the observed prevalence in the combination of CLT/GD patients (Table 3). Regarding surgery dates, subjects who underwent intervention after the first four-year period (2005-2008) had an increased risk of developing PTMc. This increase was significant in the group treated in 2017-2020, which has an OR of $2.03(\mathrm{CI} 95 \%, 1.24-3-33 ; p=0.005)$ when compared to the first four-year period. 
Table 3

Results of a Multivariate logistic regression analysis to test the association of benign thyroid pathology and surgery date.

\begin{tabular}{|llll|}
\hline & Odds ratio & $95 \% \mathrm{Cl}$ & $\mathrm{p}$ \\
\hline Multinodular goiter & 2.2 & $1.4-3.5$ & 0.001 \\
\hline Surgery date: & & & \\
$-2005-2008$ & 1 & & \\
$-2009-2012$ & 1.23 & $0.75-2.06$ & 0.424 \\
$-2013-2016$ & 1.21 & $0.72-2.04$ & 0.463 \\
$-2017-2020$ & 2.03 & $1.24-3.33$ & 0.005 \\
\hline
\end{tabular}

\section{Discussion}

PTMc is the most frequently incidentally discovered thyroid neoplasm because of its indolent and asymptomatic course (14). Prognosis of incidental PTMc is excellent, with recurrence rate of $0.5 \%$ and mortality as low as $0 \%$ (15). In this direction, it has been observed that PTMc show an alternative rearrangement gene expression pattern compared to PTC. Therefore that specific microenvironment is responsible for the different phenotypic and clinical expression (16)(17). Consequently American Thyroid Association (ATA) guidelines and the 8th AJCC/TNM system, suggest less aggressive therapeutical approaches $(18,19)$. It has even been proposed active surveillance of low-risk PTMc as the best first-line treatment, particularly in elderly (20) (21).

There are some clinical characteristics that modify this indolent evolution of PTMc, conferring a subgroup of patients a higher risk. These modifiers are youth (under 19 years), multifocality, microscopic features of aggressiveness (tall cell, blood vessel permeation), incidental diagnosis in patients with clinical metastases (6). Some authors have proposed a subdivision according to size, with a cut-off point of $7 \mathrm{~mm}$, as this seems to be related with tumorigenic behavior (22). Three different subtypes of PTMc have been identified: type I (incidentally detected PTMc with no symptoms, harmless the life expectancy), type II (accompanied by small lymph node metastasis and/or minimal invasion with no progression) and type III (high-risk for presenting data on aggressiveness) (23). Recent studies have shown a growing prevalence of PTC over the last decades (1). Our local thyroid cancer registry of the Community of Navarre (Spain) is consistent with this trend. It demonstrated an increase of TC diagnosis in both sexes, as a result of a gradual rise of T1a papillary carcinoma probably due to a higher diagnosis of microcarcinomas over the years (24). Frequency of incidentally PTMc found in surgical series differs depending on the cohort reviewed, ranging from 2-40\% (25). Interpretation of this inter-study variability could be explained by geographical factors (environmental features, iodine status, diet, medical healthcare access) and underlying pathology of the subjects that leads them to surgery. The first study that proved a grater incidental thyroid cancer risk in patients referred to surgery was carried out by Smith 
et al. (26), with an overall prevalence of $15.6 \%$. PTMc prevalence in our cohort was $9.3 \%(167 / 1802)$, which places us near the lower limit of other series (27)(28)(26)(29). A possible explanation of this results could be that there are no environmental exposures that increase risk of PTMc in our region. Exclusion criteria were strict because the main objective was to include only patients with truly incidentally discovered PTMc and avoid selection vias. Therefore, all patients with a suspicion of thyroid carcinoma confirmed by histologic examination were not included.

Our analysis found a higher prevalence of occult PTMc in MNG, followed by other benign pathologies such as CLT and finally GD. Leading indication for surgery in our center was MNG, probably due to the relatively high prevalence in our environment, which is at endemic levels (30). Moreover, thyroid nodules are the most frequent thyroid disease. The term of MNG is currently used when there are several nodules in the thyroid gland. Overall, $10.5 \%$ of the MNG operated (147/1406) had an occult microcarcinoma, similar to the $12 \%$ risk described by Fama et al (25) and $14 \%$ of Taşova et al (31), but lower than the $29.2 \%$ of the cohort of Ajarma et al (32). This percentage is comparable to pre-surgery probability of malignancy of nodules, ranging from $7-15 \%$, depending on age, sex, radiation history or family history (18).

Opposite to our findings, other authors have reported a greater prevalence of cancer in patients with underlying thyroiditis. Nevertheless, relationship between thyroid autoimmunity and cancer remains controversial. Some studies have demonstrated that there is a link between lymphocytic infiltration of the thyroid parenchyma and PTC, an histologic feature described in Hashimoto's disease (33). The pathophysiological mechanism responsible is not fully understood, however Virchow in 1863 had speculated the link between chronic inflammation and neoplastic transformation of normal tissue (34). Some of trigged mechanisms proposed for carcinogenesis are stimulation due to the action of TSH, expression of specific proto-oncogenes and chemokines produced by tissue-infiltrating lymphocytes (35). Autoimmune role of antibodies and chronic lymphocyte infiltration may predispose for dysplastic evolution of the follicular epithelium, creating a pre-neoplastic area progressing toward the existence of a tumor (36). Hashimoto's thyroiditis has been reported that only increases the risk of PTC in euthyroid individuals and in those that partially preserve the function (37). High TSH levels leads to cellular hypertrophy and hyperplasia by a constitutive activation of this pathway, tiggering genetic abnormalities. In this direction, Fiore E. et al (38), showed that risk of malignancy is associated with increased in TSH values. Microenvironment and molecular investigation of thyroid cancer is crucial because it may explain why the same histological subtype have different behavior. Research have found that PTC is less frequent and aggressive in GD as compared to CLT and non-autoimmune thyroid disease (39). Nevertheless, it has been considered that cancer and autoimmunity were extremes of immune-responses (40).

Our cohort reported one of the lowest prevalence of PTMc in CLT $(3 / 46=6.5 \%)$, compared to other series. Slijepcevic et al (29) show a different distribution of incidental PTMc in relation to the benign thyroid disease to undergoing surgery, with the highest prevalence in Hashimoto thyroiditis (22.7\%). Bircan et al (41) noted around $39 \%$. Notably, the indication for CLT surgery is the lowest of all in our cohort. This may 
be due to the strict inclusion criteria. Probably, areas of thyroiditis in the parenchyma may form nodules with an ultrasound appearance that mimics nodules with intermediate or high suspicion (42)(43). This pre-surgical suspicion could lead to perform cytological studies by fine needle aspiration biopsy (FNAB) and, if malignancy is confirmed by histological analysis of the specimen, these subjects would be excluded.

The current study found the lowest risk in GD, with a frequency of PTMc around 4.9\% (17/349). Prevalence reported range from $0.5-15 \%$, with many cohorts submitting rates below $5 \%$. Dănilă R. et al. (44), in a retrospective study performed on a consecutive 92 patients operated with GD, conclude that the $2.2 \%$ prevalence of incidental thyroid microcarcinoma was similar to other benign disease. Lower distribution of PTMc in autoimmune disorders compared to MNG, suggest that thyroid autoimmunity does not affect tumorigenesis (45).

Age was not found to affect the risk of malignancy. Our results are in concordance with Luo et al. (46) who reported that age was not a very strong independent factor for predicting malignancy (OR 0.97, $95 \% \mathrm{Cl} 0.960-0.987, \mathrm{p}<0.001)$ due to an odds ratio approached to 1 . Consequently, age is not helpful for predicting malignancy. However, it seems to influence on progression and prognosis of PTMc (21).

In our study we did not find sex as a risk factor to predict PTMc, with a male prevalence of $11.1 \%$ (36/323) compared to $17.3 \%$ (131/759) in females. Slijepcevic N. et al. (29), did not report gender differences in his cohort of 2,466 patients. Roti E. et al. (47), corroborated this same theory. This phenomenon does not seem to occur in cancers of a larger size, since the overall higher prevalence of papillary PTC in women suggests a role in promoting malignancy transformation attributable to estrogen stimulation (48). At the onset of puberty, the prevalence of PTC increases only in females, decreasing again after menopause, possibly due to the growth-promoting effect mediated by membrane-bound estrogen receptors (49).

Rising prevalence of PTMc in our cohort over decades (24), is a fact that is consistent with other series (14). Leenhardt L. et al. (50), described an increase of $8.1 \%$ and $6.2 \%$ per year in women and men, due to papillary type with an epidemic tumors measuring less than 1 centimeter ( $43 \%$ of total operated cancers). Rego-Iraeta A. et al. (51), support these results. This Spanish descriptive epidemiological study demonstrates that the increase is exclusive of PTMc. Besides PTMc, they do not identify significant variations in tumor size over time. One possible explanation that may have influenced in this increasing incidence in the population of Navarre is the change in the iodization situation. There has been a progressive change in recent decades from an iodine-deficient to an iodine-sufficient community, although the association between increased iodine intake and thyroid cancer is controversial. Another factor to take into is that the number of pathologic slides from each surgical specimen has increased over the years, contributing to the detection of PTMC specimens. Overexposure to ionizing radiation sources through the decades may also contribute to this effect.

In conclusion, we found a prevalence of $9.3 \%$ of incidental PTMc, that is comparable to rates of other European cohorts. Age and gender are not independent predictors for PTMc, with a higher prevalence of 
incidental PTMc in MNG followed by CLT and lastly in GD. Surgical findings of PTMc in total thyroidectomy for benign disease has increased significantly over the years, particularly in the 20172020 period.

\section{Declarations}

\section{Author contributions}

Dr. de Carlos was in charge of analysis, writing up the article and interpretation of data. Dr. Ernaga was responsible for overseeing the project, data collection and manuscript correction. Dr. Irigaray was responsible of data analysis, data collection and revising it critically. Dr. Pineda was responsible of follow-up patients, data acquisition and intellectual producer. Dr. Echegoyen was responsible of the histological study of the sample and study designs. Dr. Salvador was responsible of surgery, literature search and follow-up patients. Dr. Anda overall coordinator of the entire study. All authors discussed previous versions of the manuscript and agreed to the submission of the final version.

\section{Ethics approval and Consent to participate}

Ethical principles for medical research involving human subjects in accordance with the World Medical Association Declaration of Helsinki has been conducted. The study protocol has been approved by the ethics committee of the Government of Navarre (Spain). This study has been granted an exemption from requiring written informed consent by the ethics of the Government of Navarre (Spain).

\section{Acknowledgement}

The authors would like to thank the Surgery, Pathology, Radiology, Nuclear Medicine and Endocrinology Department at Hospital Universitario de Navarra for the support provided in treating patients.

The authors did not receive any funding for the present work.

\section{Conflict of Interest Statement}

All authors have completed the ICMJE uniform disclosure form. The authors have no conflicts of interest to declare.

\section{References}

1. Kitahara CM, Sosa JA. The changing incidence of thyroid cancer. Nat Rev Endocrinol [Internet]. 2016;12(11):646-53. Available from: http://dx.doi.org/10.1038/nrendo.2016.110

2. La Vecchia C, Malvezzi M, Bosetti C, Garavello W, Bertuccio P, Levi F, et al. Thyroid cancer mortality and incidence: A global overview. Int J Cancer. 2015;136(9):2187-95.

3. Lim H, Devesa SS, Sosa JA, Check D, Kitahara CM. Trends in thyroid cancer incidence and mortality in the United States, 1974-2013. JAMA - J Am Med Assoc. 2017;317(13):1338-48. 
4. Hyeong Sik Ahn, M.D., Ph.D., Hyun Jung Kim, M.P.H., Ph.D., and H. Gilbert Welch, M.D. MPH. Korea's Thyroid-Cancer “Epidemic” - Screening and Overdiagnosis. N Engl J Med. 2014;371(19):1765-7.

5. Lloyd RV, Osamura RY, Klöppel G, Rosai J E. WHO Classification of Tumours of Endocrine Organs 4.th edition Lyon: IARC; 2017.

6. Rosai J, LiVolsi VA, Sobrinho-Simoes M, Williams ED. Renaming Papillary Microcarcinoma of the Thyroid Gland: The Porto Proposal. Int J Surg Pathol. 2003;11(4):249-51.

7. Lloyd RV, Osamura RY, Klöppel G RJ. WHO Classification of Tumours of Endocrine Organs,4th Edition, Volume 10. Vol. NO. 23, IARC (International Agency for Research on Cancer) Scientific Publications. 2017. 65-142 p.

8. Elliott MS, Gao K, Gupta R, Chua EL, Gargya A, Clark J. Management of incidental and non-incidental papillary thyroid microcarcinoma. J Laryngol Otol. 2013;127 Suppl(April):17-23.

9. Bernet V. Approach to the patient with incidental papillary microcarcinoma. J Clin Endocrinol Metab. 2010;95(8):3586-92.

10. Ahmed SR, Ball DW. Incidentally discovered medullary thyroid cancer: Diagnostic strategies and treatment. J Clin Endocrinol Metab. 2011;96(5):1237-45.

11. Sapalidis K, Papanastasiou A, Michalopoulos N, Mantalovas S, Giannakidis D, Koimtzis GD, et al. A rare coexistence of medullary thyroid cancer with graves disease: A case report and systematic review of the literature. Am J Case Rep. 2019;20:1398-401.

12. Tuttle RM, Fagin JA, Minkowitz G, Wong RJ, Roman B, Patel S, et al. Natural history and tumor volume kinetics of papillary thyroid cancers during active surveillance. JAMA Otolaryngol - Head Neck Surg. 2017;143(10):1015-20.

13. Neuhold N, Schultheis A, Hermann M, Krotla G, Koperek O, Birner P. Incidental papillary microcarcinoma of the thyroid - Further evidence of a very low malignant potential: A retrospective clinicopathological study with up to 30 years of follow-up. Ann Surg Oncol. 2011;18(12):3430-6.

14. Londero SC, Krogdahl A, Bastholt L, Overgaard J, Trolle W, Pedersen HB, et al. Papillary thyroid microcarcinoma in Denmark 1996-2008: A national study of epidemiology and clinical significance. Thyroid. 2013;23(9):1159-64.

15. Domínguez JM, Nilo F, Martínez MT, Massardo JM, Muñoz S, Contreras T, et al. Papillary thyroid microcarcinoma: Characteristics at presentation, and evaluation of clinical and histological features associated with a worse prognosis in a Latin American cohort. Arch Endocrinol Metab. 2018;62(1):6-13.

16. Hsu YC, Lee JJ, Chien MN, Chen MJ, Leung CH, Cheng SP. Is papillary thyroid microcarcinoma a biologically different disease? A propensity score-matched analysis. J Surg Oncol. 2019;120(6):1023-30.

17. Rodrigues AC, Penna G, Rodrigues E, Castro P, Sobrinho-Simões M, Soares P. The Genetics of Papillary Microcarcinomas of the Thyroid: Diagnostic and Prognostic Implications. Curr Genomics [Internet]. 2017;18(3):244-54. Available from: https://www.ncbi.nlm.nih.gov/pmc/articles/PMC5476952/pdf/CG-18-244.pdf 
18. Haugen BR, Alexander EK, Bible KC, Doherty GM, Mandel SJ, Nikiforov YE, et al. 2015 American Thyroid Association Management Guidelines for Adult Patients with Thyroid Nodules and Differentiated Thyroid Cancer: The American Thyroid Association Guidelines Task Force on Thyroid Nodules and Differentiated Thyroid Cancer. Thyroid. 2016;26(1):1-133.

19. Editor D. 8th edition of the AJCC / TNM staging system of thyroid cancer: what to expect ( ITCO \# 2 ). 2018;8-12.

20. Miyauchi A, Ito $\mathrm{Y}$, Oda H. Insights into the Management of Papillary Microcarcinoma of the Thyroid. Thyroid. 2018;28(1):23-31.

21. Ito Y, Miyauchi A, Kihara M, Higashiyama T, Kobayashi K, Miya A. Patient age is significantly related to the progression of papillary microcarcinoma of the thyroid under observation. Thyroid. 2014;24(1):27-34.

22. Lee KJ, Cho YJ, Kim SJ, Lee SC, Kim JG, Ahn CJ, et al. Analysis of the clinicopathologic features of papillary thyroid microcarcinoma based on 7-mm tumor size. World J Surg. 2011;35(2):318-23.

23. Sugitani I, Toda K, Yamada K, Yamamoto N, Ikenaga M, Fujimoto Y. Three Distinctly Different Kinds of Papillary Thyroid Microcarcinoma should be Recognized: Our Treatment Strategies and Outcomes. World J Surg. 2010;34(6):1222-31.

24. Rojo Álvaro J, Bermejo Fraile B, Menéndez Torre E, Ardanaz E, Guevara M AAE. Aumento de la incidencia de cáncer de tiroides en Navarra. Evolución y características clínicas, 1986-2010. Endocrinol Diabetes Nutr. 2017;64(6):303-9.

25. Fama F, Sindoni A, Cicciu M, Polito F, Piquard A, Saint-Marc O, et al. Preoperatively undiagnosed papillary thyroid carcinoma in patients thyroidectomized for benign multinodular goiter. Arch Endocrinol Metab. 2018;62(2):139-48.

26. Smith JJ, Chen X, Schneider DF, Broome JT, Sippel RS, Chen H, et al. Cancer after thyroidectomy: A multi-institutional experience with 1,523 patients. J Am Coll Surg. 2013;216(4):571-7.

27. Miccoli P, Minuto MN, Galleri D, D’Agostino J, Basolo F, Antonangeli L, et al. Incidental thyroid carcinoma in a large series of consecutive patients operated on for benign thyroid disease. ANZ J Surg. 2006;76(3):123-6.

28. Agarwal S, Agarwal A, Chand G. Incidental papillary microcarcinoma of the thyroid-further evidence of a very low malignant potential: A retrospective clinicopathologic study with up to 30 years of follow-up. Ann Surg Oncol. 2011;18(SUPPL. 3):306-306.

29. Slijepcevic N, Zivaljevic V, Marinkovic J, Sipetic S, Diklic A, Paunovic I. Retrospective evaluation of the incidental finding of 403 papillary thyroid microcarcinomas in 2466 patients undergoing thyroid surgery for presumed benign thyroid disease. BMC Cancer. 2015;15(1):1-8.

30. Caro AJM, Saura PBC, Orta JD, Rodríguez EB, Galdón MR, Gómez A. Prevalencia de bocio y deficiencia de yodo en población escolar de una zona básica de salud tradicionalmente endémica. Aten Primaria [Internet]. 2001;27(4):258-62. Available from: http://dx.doi.org/10.1016/S0212-6567(01)78805-1 
31. Tasova V, Kilicoglu B, Tuncal S, Uysal E, Sabuncuoglu MZ, Tanrikulu Y, et al. Evaluation of incidental thyroid cancer in patients with thyroidectomy. West Indian Med J. 2013;62(9):844-8.

32. Ajarma KY, Al-Faouri AF, Al Ruhaibeh MK, Almbaidien FA, Nserat RT, Al-Shawabkeh AO, et al. The risk of thyroid carcinoma in multinodular goiter compared to solitary thyroid nodules: A retrospective analysis of 600 patients. Med J Armed Forces India [Internet]. 2020;76(1):23-9. Available from: http://dx.doi.org/10.1016/j.mjafi.2018.05.001

33. Graceffa G, Patrone R, Vieni S, Campanella S, Calamia S, Laise I, et al. Association between Hashimoto's thyroiditis and papillary thyroid carcinoma: A retrospective analysis of 305 patients. BMC Endocr Disord. 2019;19(Suppl 1):4-9.

34. R V. Standpoints in Scientific Medicine. Bull Hist Med. 1877;30:537-543.

35. Vita R, leni A, Tuccari G, Benvenga S. The increasing prevalence of chronic lymphocytic thyroiditis in papillary microcarcinoma. Rev Endocr Metab Disord. 2018;19(4):301-9.

36. Chui MH, Cassol CA, Asa SL, Mete O. Follicular epithelial dysplasia of the thyroid: Morphological and immunohistochemical characterization of a putative preneoplastic lesion to papillary thyroid carcinoma in chronic lymphocytic thyroiditis. Virchows Arch. 2013;462(5):557-63.

37. Paparodis R, Imam S, Todorova-Koteva K, Staii A, Jaume JC. Hashimoto's thyroiditis pathology and risk for thyroid cancer. Thyroid. 2014;24(7):1107-14.

38. Fiore E, Vitti P. Serum TSH and risk of papillary thyroid cancer in nodular thyroid disease. J Clin Endocrinol Metab. 2012;97(4):1134-45.

39. Imam S, Dar P, Paparodis R, Almotah K, Al-Khudhair A, Hasan SAM, et al. Nature of coexisting thyroid autoimmune disease determines success or failure of tumor immunity in thyroid cancer. $J$ Immunother Cancer. 2019;7(1):1-13.

40. Romero P, Dunbar PR, Valmori D, Pittet M, Ogg GS, Rimoldi D, et al. Ex vivo staining of metastatic lymph nodes by class I major histocompatibility complex tetramers reveals high numbers of antigenexperienced tumor-specific cytolytic T lymphocytes. J Exp Med. 1998;188(9):1641-50.

41. Bircan HY, Koc B, Akarsu C, Demiralay E, Demirag A, Adas M, et al. Is Hashimoto's thyroiditis a prognostic factor for thyroid papillary microcarcinoma? Eur Rev Med Pharmacol Sci. 2014;18(13):1910-5.

42. Yildirim D, Gurses B, Gurpinar B, Ekci B, Colakoglu B, Kaur A. Nodule or pseudonodule? differentiation in Hashimoto's thyroiditis with sonoelastography. J Int Med Res. 2011;39(6):2360-9.

43. Anderson L, Middleton WD, Teefey SA, Reading CC, Langer JE, Desser T, et al. Hashimoto thyroiditis: Part 2, sonographic analysis of benign and malignant nodules in patients with diffuse Hashimoto thyroiditis. Am J Roentgenol. 2010;195(1):216-22.

44. Dănilă R, Karakas E, Osei-Agyemang T, Hassan I. Outcome of incidental thyroid carcinoma in patients undergoing surgery for Graves' disease. Vol. 112, Revista medico-chirurgicală a Societăţii de

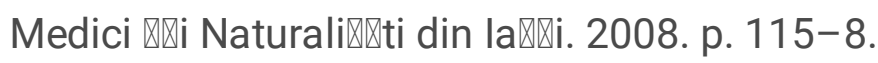

45. Pacini F, Mariotti S, Formica N, Elisei R, Anelli S, Capotorti E PA. Thyroid autoantibodies in thyroid cancer: incidence and relationship with tumour outcome. Acta Endocrinol. 119(3):373-80. 
46. Luo J, McManus C, Chen H, Sippel RS. Are there predictors of malignancy in patients with multinodular goiter? J Surg Res. 2012;174(2):207-10.

47. Roti E, Rossi R, Trasforini G, Bertelli F, Ambrosio MR, Busutti L, et al. Clinical and histological characteristics of papillary thyroid microcarcinoma: Results of a retrospective study in 243 patients. J Clin Endocrinol Metab. 2006;91(6):2171-8.

48. Derwahl M, Nicula D. Estrogen and its role in thyroid cancer. Endocr Relat Cancer. 2014;21(5):T27383.

49. Zane M, Parello C, Pennelli G, Townsend DM, Boscaro M, Toniato A, et al. Estrogen and thyroid cancer is a stem affair: A preliminary study. Biomed Pharmacother. 2017;85:399-411.

50. Leenhardt L, Grosclaude P C-CL. Thyroid Cancer Committee. Increased incidence of thyroid carcinoma in france: a true epidemic or thyroid nodule management effects? Report from the French Thyroid Cancer Committee. Thyroid. 12:1056-60.

51. Rego-Iraeta A, Pérez-Méndez LF, Mantinan B G-MR. Time trends for thyroid cancer in northwestern Spain: true rise in the incidence of micro and larger forms of papillary thyroid carcinoma. Thyroid. 19 (4):333-40.

\section{Figures}




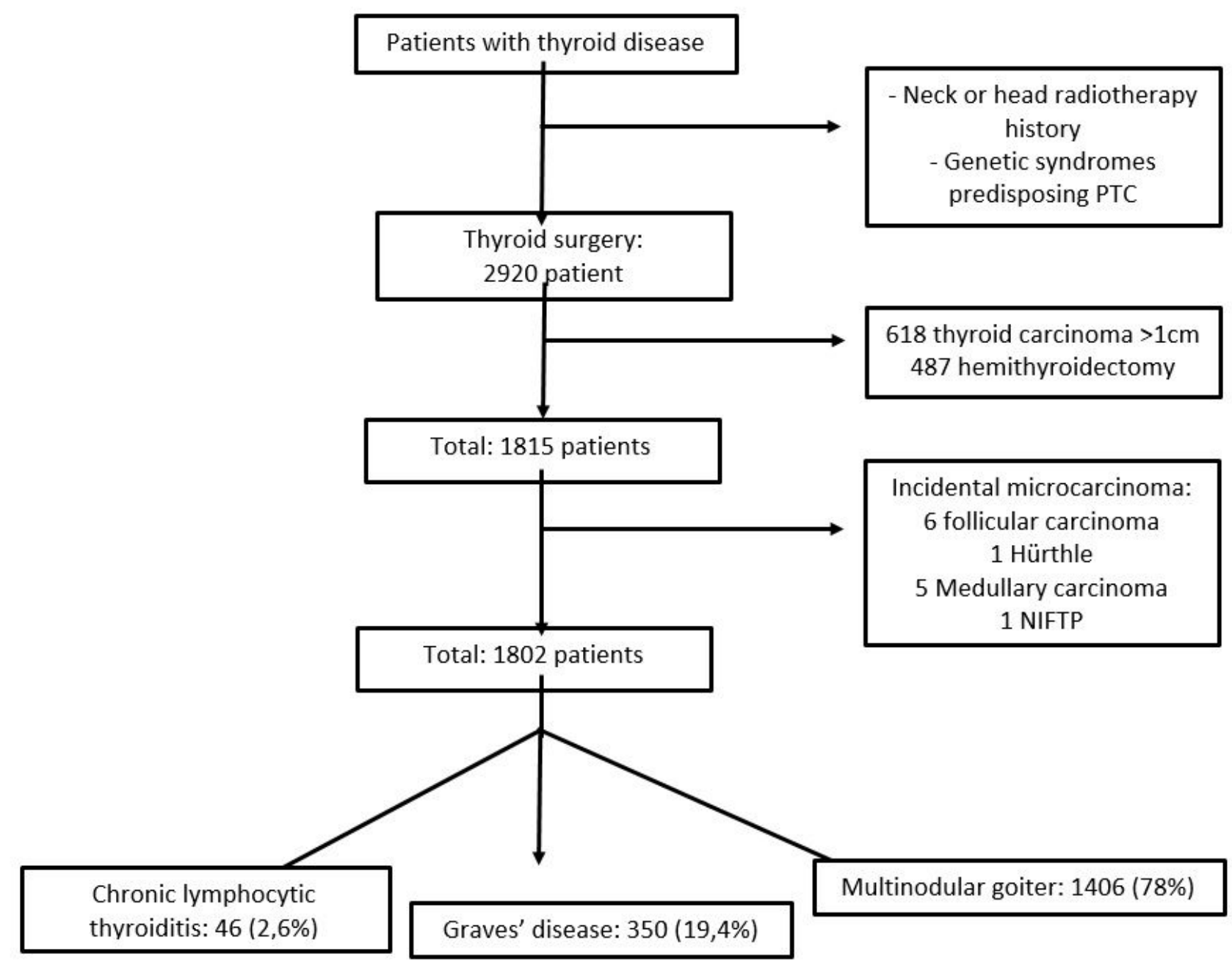

Figure 1

Flow chart for the selection of patients, with inclusion and exclusion criteria.

NIFTP, Non-invasive follicular thyroid neoplasm with papillary like nuclear features. 


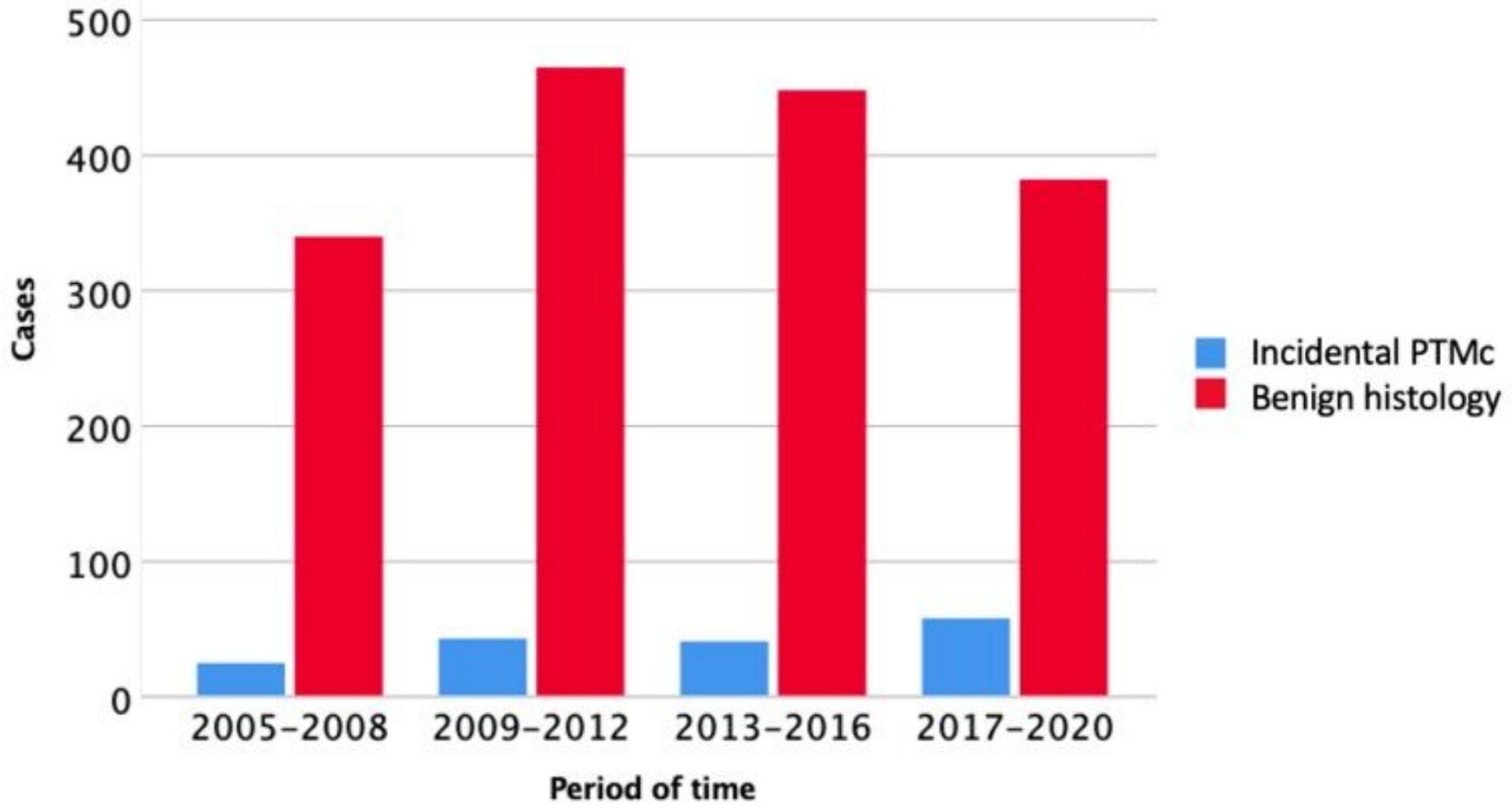

Figure 2

Total surgeries, cases of papillary thyroid microcarcinoma and benign histology. 